\title{
Le terrorisme comme fiction sociale : retour sur l'affaire Nasr Hâmid Abu Zayd
}

\author{
Amine Mohamed Brahimi \\ École des hautes études en sciences sociales
}

\section{Le terrorisme comme fiction sociale : retour sur l'affaire Nasr Hâmid Abu Zayd}

Le terrorisme a été, durant les cinquante ans de la guerre froide, un jeu aux règles clairement établies. Avec la chute du mur de Berlin (1989), cette violence politique s'est déstructurée, au point de contaminer des entités jusque-là éloignées de telles pratiques. Les nouveaux acteurs qualifiés de terroristes sont désormais plus imprévisibles, et leur violence a pris une dimension beaucoup moins ordonnée idéologiquement. En parallèle à cette transformation profonde, il est aisé de constater la multiplication des discours autour de la question du terrorisme ${ }^{1}$. Souvent appréhendé comme une catégorie abstraite et homogène, le terrorisme renvoie à des schémas explicatifs linéaires. Cette disposition d'esprit place souvent le complot islamiste au centre de toute volonté explicative. Elle occulte fréquemment une réalité sociale beaucoup plus complexe. Le but du présent texte est de déconstruire ce type de discours à l'aide des outils fournis par les sciences sociales.

Notre démarche a pour objectif de caractériser la fiction sociale telle qu'elle est produite par la question terroriste et la manière dont ce dispositif participe à effacer les multiples enjeux sociaux soulevés par ce type de violence politique. Pour illustrer ce fait, notre choix s'est porté sur l'affaire Nasr Hâmid Abu Zayd, à la fois comme cas d'étude, mais aussi comme un indicateur des différents déterminants qui peuvent précéder la construction sociale de la menace terroriste ${ }^{2}$.

\section{La fiction comme construction sociale}

La référence fictionnelle est souvent liée à la production artistique. La fiction romanesque ou ludique fait appel généralement à un processus narratif autonome et donne naissance à un ensemble d'univers imaginaires. Se distançant souvent de tout rapport à la vérité, ce type d'œuvre reflète une série de situations virtuelles à explorer. Le présent article aborde l'objet

\footnotetext{
${ }^{1}$ Voir sur le sujet le numéro spécial de la revue Topique « Représentations du terrorisme» (de Mijolla-Mellor et Kiss).

${ }^{2}$ Ce texte résulte d'une recherche de terrain effectuée au Caire (Égypte) durant le mois de septembre 2016. Dans ce cadre, nous avons effectué plusieurs entretiens avec les proches de Abu Zayd, notamment sa femme.
} 
fictionnel comme partie prenante d'un construit social (Flahault et Heinich). Par opposition à la fiction artistique, ce type de récit entretient un rapport étroit à la notion de vérité. En effet, la fiction sociale fait partie d'un discours indexé sur la réalités.

Cette illusion cognitive devient alors un filtre qui modèle notre perception du réel en dehors de notre attention consciente (Heinich et Schaeffer 163-186). La fiction sociale évolue de ce fait, en une dimension fondatrice dans les perceptions à l'égard de certains phénomènes. Le discours fictionnel a donc vocation à guider la perception des acteurs sociaux. On se rapproche ainsi de l'« idée régulatrice » kantienne dont le but premier est de servir d'horizon à la pensée (Kant 77). Faisant office d'automatisme intellectuel, la fiction sociale produit alors un certain nombre de représentations erronées sur la société. Ce type de fiction n'est donc jamais appréhendé comme fiction; sa corrélation à l'ordre réel est un élément essentiel pour garantir sa performativité (Hume et Malherbe 74).

Le point central de notre réflexion sera de démontrer comment le terrorisme peut réduire notre compréhension de la réalité sociale des pays à majorité musulmane. La menace terroriste semble souvent subsumer toute analyse sur cette région au point de créer une fiction sociale qui réduit toute tentative explicative. Ce genre d'illusion participe à travers l'exagération du « danger terroriste » à brouiller toute tentative d'explication complexe relative à ce phénomène social. De plus, la présence exagérée de cette fiction sociale dans les médias, lorsqu'on parle des sociétés musulmanes, a pour conséquence d'essentialiser la vie politique et intellectuelle de ces sociétés. Cette simplification semble alors servir à la fois une vision de l'islam comme source de toutes les formes d'engagement politique dans les mondes musulmans ${ }^{4}$; mais aussi une opposition idéologique binaire entre un islam dit « libéral » ou «progressiste » et un islam «fondamentaliste ${ }^{5}$. Le terrorisme serait à la fois un "mot fédérateur » au sens qu'il regroupe, à travers le même schème explicatif, plusieurs expériences variées; et un motécran qui, sous l'apparence d'une seule causalité, tend à occulter la complexité des relations sociales qui lient les champs politique, académique et intellectuel. Cette utilisation du terme terrorisme atteint sa plus grande efficacité quand elle prend comme véhicule, les moyens de communication de masse. Ces moments médiatiques permettent d'implémenter un «cadrage» (framing) spécifique (Goffman).

\footnotetext{
3 Ce constat autour de la fiction doit être relativisé notamment à propos de toute une littérature où s'entremêlent fiction et récits autobiographiques. Voir sur le sujet: Signes de vie. Le pacte autobiographique 2 (Lejeune).

${ }^{4}$ Quoiqu'il ne s'agisse pas de l'objet principal du texte, l'origine de ce type de représentation a été explicitée dans les recherches qui traitent de l'orientalisme, voir à ce sujet : (Said; AbdelMalek; Abaza et Stauth).

${ }^{5}$ Pour une perspective binaire de la pensée progressiste en islam, voir (Benzine); pour mieux cerner ce type de démarche, voir en ce qui concerne l'islamisme : (Ayubi; Kepel) .
} 
Ce dispositif, par lequel s'effectue la promotion d'une définition spécifique du problème (par-là énoncé), son discernement, son appréciation morale et/ou son procédé, s'opère par le biais de représentations ancrées d'une réalité telle qu'appréhendée par le sens commun; particulièrement de certains de ses éléments présentés comme proéminents. La focalisation se fera sur cette seule « réalité » en tant qu'entité désignée. Tout ce qui gravitera autour sera éclipsé, du moins inexistant pour le public (Kahneman et Tversky).

Les journalistes jouent un rôle important au niveau de ce cadrage des nouvelles. En effet, il existe un lien étroit entre leur manière d'opérer et leurs habitudes sociales et professionnelles. La pratique du cadrage et son élaboration sont interprétées par l'interdépendance entre les règles et procédés des journalistes avec l'emprise telle qu'accomplie par des groupes d'intérêts (Scheufele; Gamson et Modigliani).

En se basant sur une impitoyable logique économique, par contraintes budgétaires ou par nécessité de maximiser les profits, les institutions télévisuelles (publiques ou privées) vont privilégier la prééminence des sensationnalistes (Noam et Brousseau). On a affaire alors à « un mercantilisme des médias et leur soumission de plus en plus débridée, dans le cadre d'une concurrence exacerbée, au marketing comme loi d'airain de la satisfaction du public à tout prix : l'information s'alignerait de plus en plus sur ce que le public veut savoir plutôt que sur ce que le public doit savoir $»$ (Demers 1).

En plus d'élaborer le cadrage nécessaire pour percevoir l'actualité immédiate, l'information participe en même temps à la création d'une perception spécifique. Ainsi, l'actualité du moment, celle du sensationnel qui suscite l'émotion est régulièrement mise à contribution, engendrant par-là les opinions détachées d'un quelconque raisonnement. La fabrique quotidienne des représentations est de ce fait déclenchée, imprégnant les mentalités d'une fiction sociale désormais ancrée, au détriment d'une réalité totalement occultée et d'une expérience humaine en latence.

Dans cette optique, cette conception fictionnelle, souvent totalisante, met en scène des récits fabriqués impliquant leurs principaux acteurs dans des situations et des actions représentées. C'est le cas de l'islamisme politique ${ }^{6}$, qui n'offre aucun recul par rapport à l'événement et met fin momentanément à tout commentaire à ce sujet; ceci indépendamment de toute validité ou tout démenti via les événements réels en lien. Ce sont les présupposés politiques implicites, les effets désastreux

${ }^{6}$ La médiatisation qui a suivi les événements du 11 septembre 2001 est un bon exemple de cette vision fictionnelle, voir : (Lits). 
sur l'information ainsi que ces manœuvres fictives que nous nous proposons de démêler (Tétu).

\section{L'intellectuel et le terrorisme : l'affaire Hâmid Nasr Abu Zayd}

Pour mieux appréhender la fiction sociale que peut créer une menace terroriste, nous avons choisi de nous concentrer sur les effets de la violence extrémiste dans le champ intellectuel, à travers l'analyse de l'affaire Nasr Hâmid Abu Zayd. Les mésaventures de ce professeur de littérature égyptien ont marqué l'opinion internationale durant les années 1990. Faisant face à des menaces terroristes, Abu Zayd sera obligé de quitter Le Caire. L'ambiance politico-intellectuelle égyptienne de l'époque semble alors hostile aux discours critiques sur la religion musulmane ${ }^{7}$. En effet, l'affaire Nasr Hâmid Abu Zayd se situe dans un contexte caractérisé par une série d'attentats visant des intellectuels : le polémiste Farag Foda a été assassiné en 1993. Une année plus tard, le prix Nobel de littérature 1988 Naguib Mahfouz était poignardé par un islamiste. Cette attaque handicape l'écrivain, devenu incapable d'utiliser sa main pour écrire (Fayek), sans compter qu'en 1997, le philosophe Hassan Hanafi a reçu des menaces de mort à la suite d'une conférence sur la pensée islamique, sous prétexte d'apostasie (Shaping Global Islamic Discourses 73). C'est dans cet environnement qu'a commencé l'affaire Abu Zayd, par un simple refus de sa demande de titularisation pour le poste de professeur. En mai 1992, les publications académiques du Dr Abu Zayd ont fait l'objet d'un examen minutieux et approfondi par l'Université du Caire. En effet, le comité permanent chargé de la titularisation et de la promotion avait été chargé de statuer sur sa demande, sur la base de ses publications. Composé de trois membres, celui-ci avait produit trois rapports. Deux d'entre eux furent en faveur du demandeur ; le troisième, émanant du professeur en philologie arabe Abdel-Sabour Shahin ${ }^{8}$, émit quant à lui, un avis défavorable, avançant ainsi l'argument qu'Abu Zayd portait atteinte par ses écrits à la foi musulmane (Najjar). Le comité s'aligna au final sur cette décision en votant majoritairement (sept voix contre six) contre la demande d'Abu Zayd, jugeant ainsi que ses travaux ne justifiaient nullement une quelconque titularisation. Cette décision ne manqua pas d'être critiquée par le Conseil du Département arabe ainsi que par le Conseil de la Faculté des Lettres. Malheureusement, cela n'empêcha pas le Conseil de l'Université du Caire de confirmer la décision du Comité permanent le 18 mars 1993, en rejetant la demande de titularisation d'Abu Zayd (Bakr, Colla, et Zayd).

\footnotetext{
${ }^{7}$ Les discours critiques sur la religion musulmane ont souvent créé des controverses en Égypte, et ce, depuis le début du 20e siècle (Taha Hussein, Abderraziq) voir : (Adams). La particularité des années 1990 se situe dans la nature violente de ce type de controverse.

${ }^{8}$ Quelques années après l'affaire Abu Zayd, Shahin a lui-même subi la colère de l'Académie des recherches islamiques d'Al Azhar : elle a jugé son livre «Abi Adam » peu conforme au dogme islamique sur la genèse et la Création.
} 
La médiatisation de cette affaire a eu des effets néfastes sur Abu Zayd, lequel s'est vu assigné d'une plainte portée par un juriste réclamant son divorce de sa femme, la Dr Ibthal Younis. Soutenue par le fait qu'une femme musulmane ne peut être mariée avec un présumé apostat, cette requête fut rejetée par la Juridiction inférieure sur le Statut personnel de Giza le 27 janvier 1994, le plaignant n'ayant pas d'intérêt direct ni personnel (Sfeir). Malgré cela, elle eut quand même gain de cause par la cour d'appel du Caire qui lui donna une suite favorable et annula le mariage d'Abu Zayd et Ibtihal Younes en 1995. Alors qu'entre temps (deux semaines avant ce jugement), le professeur avait obtenu sa titularisation avec les félicitations du Comité, l'une des conséquences du verdict de la cour d'appel ne tarda pas : le groupe islamiste armé 'Jihad' (qui assassina le président égyptien Anouar el-Sadate en 1981) menaça de mort Abu Zayd en énonçant clairement son exécution imminente, justifiant cette décision par l'abandon supposé de la foi musulmane. Malgré la protection reçue des forces policières, Nasr Hâmid Abu Zayd, en compagnie de son épouse, décida de quitter Le Caire pour s'installer aux Pays-Bas, le 23 juillet 1995. Il obtint d'ailleurs le poste de professeur à l'Université de Leyde puis, la direction de la Chaire Ibn Rushd Humanisme et Islam à l'Université d'Utrecht (Ferrié et Dupret).

La menace terroriste sur laquelle se concluent les événements de l'affaire Nasr Hâmid Abu Zayd peut servir de fiction sociale au sens qu'elle justifierait une lecture superficielle de cet événement. En effet, la simple opposition en termes de valeurs ou de perception entre deux visions de l'islam n'est pas suffisante. Loin de nier la réalité de la menace terroriste, il s'agit d'appréhender cet événement à travers les multiples enjeux sociaux qui le sous-entendent. L'affaire Nasr Hâmid Abu Zayd se comprend donc comme un cas dans le sens où elle nous pousse à interroger nos a priori : elle appelle à " l'instauration d'un cadre nouveau du raisonnement» (Passeron et Revel 10). Ce cas singulier devient alors un moyen d'éclairer des problèmes beaucoup plus généraux. Nous avons choisi deux aspects qui nous semblent centraux dans cette affaire et qui démontrent à quel point elle dépasse le simple «cadre » du terrorisme : les rivalités universitaires qui sousentendent l'affaire Nasr Hâmid Abu Zayd et le jugement qui a suivi son refus de promotion.

\section{Discours religieux et autonomie académique}

La vision d'Abu Zayd concernant l'islam et les références au Coran et textes sacrés a évolué tout au long de sa trajectoire universitaire, et ce en tendant vers une perspective contextuelle. Elle se structure autour d'une interprétation des écrits replacés dans leur espace sociopolitique immédiat aussi bien sur le plan historique, que linguistique et culturel (Taji-Farouki 169). 
Son ultime reproche à l'encontre des érudits, plus fréquemment les élites des sociétés musulmanes, concerne leur instrumentalisation autant intellectuelle que politique du Coran. Selon lui, leur erreur est de voir un texte dans le livre sacré, alors que la majorité de la population le perçoit comme un discours (Campanini). Pour Abu Zayd, cette considération a engagé ces érudits, traditionalistes ou réformistes, dans une course, une concurrence de citations choisies dans les textes sacrés selon leur obédience respective, classifiant ainsi les extraits cités comme éclairés ou ambivalents d'après un sens attribué par les uns et les autres. Cette situation a incité les savants musulmans, conservateurs ou libéraux, à une exégèse totalitaire (Zayd).

À l'opposé de cette perspective, Nasr Hâmid Abu Zayd préconise une lecture remaniée du texte selon une exégèse humaniste, valorisant une interprétation du Coran comme discours vivant et contextualisé. Dans cette optique, il considère que le Coran peut être le produit de débats, donc d'acceptation et de rejet : une interprétation libérale pouvant ainsi offrir de nouvelles possibilités sur l'islam et provoquer d'éventuelles transformations dans les sociétés musulmanes (Zayd 93).

À partir d'une approche multidisciplinaire où s'entremêlent théologie, philosophie, droit et science politique, Abu Zayd se voulait l'instigateur d'une pensée musulmane contemporaine. Il ambitionnait d'atteindre à travers ses recherches, une forme d'exégèse humaniste qui permettrait aux musulmans d'allier, avec un changement de registre, traditions issues de la religion musulmane et pensée moderne promouvant les droits de l'homme, la liberté d'expression, la justice sociale, l'égalité entre homme et femme ainsi que la démocratie dans un contexte de mondialisation à affronter (Zayd 93). Il contribua ainsi à la mise en place d'une nouvelle littérature islamique qui postule l'existence d'un État islamique civique plutôt que religieux (Zayd 98).

C'est au-delà du simple contenu de ses recherches qu'il faut comprendre la non-titularisation du chercheur : en la liant à un contexte politique, social et intellectuel plus large. Car l'affaire Abu Zayd doit être appréhendée dans la perspective des débats post-indépendance sur la réforme religieuse. L'approche d'Abu Zayd, forte d'un cadre théorique renouvelé, s'inscrit dans une volonté de modernisation qui résulte en partie d'un contexte sociopolitique particulier. En effet, l'autonomisation partielle des champs intellectuels et religieux en Égypte est à l'origine de polémiques autour des questions de représentation du sacré. Ces "conflits herméneutiques » (Arkoun 1985) exemplifient combien l'économie des biens de salut se meut désormais entre trois sphères (politique, religieuse et

${ }^{9}$ L'ouvrage d'Ali Abderraziq, l'islam et les fondements du pouvoir, ferait aujourd'hui encore figure d'illustration exemplaire de ce processus. Voir: (Filali-Ansary; Abderraziq, al-Rāziq, et Filali-Ansary). 
intellectuelle), source de fortes conflictualités. Dans les pays à majorité musulmane, les espaces sociaux dans lesquels est mobilisé le référent religieux se structurent autour d'une «censure croisée » (Bourdieu) que les agents respectifs exercent pour affirmer leurs positions et s'opposer les uns aux autres (les modernistes dénonçant l'obscurantisme des islamistes, ces derniers stigmatisant les oulémas, etc.) (Roussillon 15). Ce rejet réciproque est surdéterminé par le contexte autoritaire des États postcoloniaux. En résulte une très forte idéologisation des discours religieux dans un contexte de réduction des libertés politiques, qui tend à annihiler les processus d'autonomisation relative des champs intellectuel et religieux, et à réduire du même coup les possibilités de dialogue entre les différents acteurs de ces espaces sociaux.

Cette ambiance de forte politisation a en grande partie influé sur la décision du comité, le rapport négatif étant vivement soutenu par un acteur (Abdel Sabour Shahin) impliqué dans le scandale des sociétés islamiques d'investissement ${ }^{10}$. Nasr Hâmid Abu Zayd avait auparavant fait une critique de ce type d'entreprise, le dénonçant dans le fait qu'il utilisait un recours à l'argumentaire religieux pour justifier une démarche frauduleuse (Zayd et Nelson 13). L'exposé général du rapport remet en compte la foi d'Abu Zayd, plutôt que sa méthodologie ou sa démarche scientifique. En effet, le manque d'autonomie du monde intellectuel pousse les acteurs académiques à être subordonnés à des enjeux extérieurs. Ainsi, Abdel Sabour Shahin est un acteur multipositionné; il occupe des positions dans les champs politique, académique et religieux. Il est imam de mosquée, professeur d'université, intellectuel public et conseiller pour le ministère des Affaires religieuses. Sa légitimité intellectuelle est indissociable d'une position politique et religieuse. Il fait donc intervenir un argumentaire avec lequel il s'interroge sur la religiosité d'Abu Zayd. Ce type d'intervention reflète un manque d'autonomie du champ intellectuel, en ce sens que des arguments extérieurs à la logique scientifique sont mobilisés pour juger du travail d'Abu Zayd. Il semble que, plus qu'à deux régimes de valeurs compétitifs, nous ayons affaire à des normes religieuses mobilisées dans des espaces sociaux qui ne leur sont pas corrélés. L'imbroglio qui sous-entend le contenu du rapport présenté Abdel Sabour Shahin peut être interprété comme un reflet de cette censure réciproque qui caractérise le champ intellectuel égyptien. L'argumentaire que propose Abu Zayd semble irrecevable, car il offre des outils d'analyse concurrents à l'orthodoxie religieuse.

\section{Cadre juridique : notion de hisba et hybridité juridique}

\footnotetext{
${ }^{10}$ Les sociétés islamiques d'investissement sont des compagnies qui ont émergé à la fin des années 1980. Elles avaient pour but de permettre aux gens d'investir leur argent et de le faire fructifier dans un cadre islamique. Ces sociétés ont été responsables de plusieurs scandales d'ordre financier (Zubaida); l'implication de Shahin au sein de ces entreprises était d'ordre public, il faisait partie des théologiens qui avaient servi de caution morale.
} 
La médiatisation de l'affaire Nasr Hâmid Abu Zayd et son débordement de l'enceinte de l'Université ont entraîné un enchaînement d'événements plutôt inattendus. Le 16 mai 1993, Abu Zayd s'est vu assigné d'une plainte déposée contre lui par un groupe d'avocats, auprès du tribunal de première instance (section du contentieux du statut personnel); celui-ci réclamait son divorce de sa femme, la Dr Ibthal Younis, comme conséquence de l'apostasie unanimement admise en jurisprudence. Cette inculpation était ainsi soutenue par le fait que sa femme, Égyptienne de confession musulmane, ne pouvait en aucun cas demeurer mariée avec un présumé apostat dont les publications affichaient certains fragments considérés comme impies, ce qui entrainerait son retrait automatique de l'islam (Blair).

La décision de la cour d'appel dans le but d'annuler le mariage d'Abu Zayd avec Ibtihal Younes était construite sur la base de la requête en hisba (Ferrié et Dupret). Non coranique, la bisba désigne, d'un côté, l'obligation pour chaque musulman d'ordonner le bien et d'interdire le mal et, de l'autre, elle balise l'office du mubtasib, inspecteur des marchés et gardien de l'ordre public. Le bien est établi par la réalisation ou la défense des droits de Dieu ; et le vigile de ces droits n'attend pour seule récompense que celle de Dieu dans l'au-delà (Johansen, 1981, p. 289-301). Traditionnellement, la bisba rendait possible pour tout musulman de briguer des recours à l'encontre de toute personne qui avait porté atteinte à l'islam, au nom de la défense des droits de Dieu (Cahen et Talbi). Dans son assise, la hisba constitue un des préceptes de l'Islam les plus importants ${ }^{11}$, celui d'être en totale conformité avec les lois de Dieu. Cette règle puise son fondement dans le Coran : « Encouragez ce qui est bon, et interdisez ce qui est mauvais » (Ferrié et Dupret 766). La hisba est structurée autour du principe de "communauté" aux fins de développer chez les croyants une même attitude, basée sur la défense des principes religieux; ce procédé est mis en œuvre dans une logique de responsabiliser chacun à contrecarrer tout acte jugé détestable et à ordonner ce qui est estimé acceptable pour la bienveillance et les intérêts généraux de la communauté ${ }^{12}$. À noter qu'avant 1996, cette procédure (soutenue par l'article 280 du règlement d'organisation des tribunaux religieux de 1931) avait été jugée recevable et applicable par certains juges. Et ce, malgré les remaniements juridiques annulant les tribunaux religieux à partir de 1956 et ordonnant leur substitution par de nouvelles instances juridiques nationales (Bälz 137). La hisba disconvenait cependant à l'article 3 du Code de procédure civile (ainsi que l'article 12 de la loi sur le Conseil d'État) en vertu duquel toute action intentée en justice n'était recevable que si le demandeur apportait la preuve

\footnotetext{
${ }^{11}$ Ici nous pensons particulièrement à l'école juridique Shâficite.

${ }^{12} \mathrm{Il}$ est nécessaire de distinguer le commandement moral (« ordonner le bien et mettre en garde contre le mal») de la hisba, en tant que notion et pratique juridique. L'ordre divin de «mettre en garde contre le mal » n'a pas toujours signifié le droit de bisba pour tous.
} 
irréfutable d'un intérêt direct et personnel à l'affaire en question (Thielmann).

La défense d'Abu Zayd fut structurée, entre autres, autour du défaut d'intérêt direct des requérants à la cause, ce qui ne manqua pas de présenter alors cette règle de droit comme étant spécifique au monde musulman, attestant de surcroît de sa non-conformité avec la conception moderne de l'ordre public. Ayant institué la légalité du recours en hisba en droit égyptien, la cour s'est alors attelée à établir l'apostasie; le but étant d'apporter la preuve que tous les discours d'Abu Zayd font de lui un apostat. Estimant qu'il y avait unanimité des ulémas et imams musulmans à ce sujet, la cour considéra qu'en tant que professeur de langue arabe et d'études islamiques, son cas était davantage aggravé par l'influence abusive qu'il exerçait supposément sur ses étudiants ; ces derniers assimilant des contenus jugés souillés par des descriptions obscènes qu'il façonnait du Coran, livre sacré de Dieu et de la Sunna de Son Prophète (Bälz 137).

Cette décision de la cour d'appel fut en fait partiellement basée sur une différenciation entre droit procédural et droit substantiel. En effet, elle était soutenue par un principe de droit procédural souscrit à la garantie de l'intérêt direct par la hisba ; celle-ci étant administrée par des orientations multiples d'ordre juridique, civil et commercial (Ferrié et Dupret 773).

C'est suite au débat intense sur des questions juridiques tel que provoqué par l'affaire Abu Zayd et pour limiter ce décuplement du nombre des requêtes en hisba déposées par des juristes proches des milieux islamistes contre des intellectuels, écrivains ou journalistes - notamment l'universitaire Nasr Hâmid Abu-Zayd (Ferrié et Dupret), le réalisateur Youssef Chahine (Bernard-Maugiron), ou encore le Prix Nobel Nagîb Mahfûz (Johansen) que le législateur égyptien a adopté en janvier 1996, la loi "portant organisation des procédures de requête directe en hisba en matière de statut personnel » (Bälz 136). L'article 1 de cette loi précise que c'est uniquement le Parquet général qui est chargé de la bisba, lui conférant à lui seul l'habilité à intenter des procès liés au statut personnel et relevant de la bisba. Le requérant qui a déposé une demande devant le Parquet général se trouvant de fait empêché de devenir partie au procès, c'est le Parquet qui décide éventuellement (après avoir auditionné les parties concernées par l'action et effectué une investigation supplémentaire) de la suite de toute requête, et qui devient lui-même requérant.

Malheureusement, cette restructuration juridique n'a pas été prise en compte dans le dossier d'Abu Zayd puisque la Cour de cassation a refusé son application sous l'argument que sa promulgation avait été opérée après l'audition de sa cause ; soit, sans appliquer d'effet rétroactif, les lois égyptiennes demeurant largement d'inspiration française (Ferrié et Dupret 773). Par ailleurs, rappelons que la Cour de cassation du Caire ne constitue 
pas un troisième degré d'instance juridique de juridiction ; elle ne juge donc pas le procès ou les décisions prononcés en dernier ressort par les juridictions du premier degré ou par les cours d'appel, lorsque ces décisions font l'objet d'un recours ou pourvoi. Son rôle n'est pas non plus de rejuger les affaires. Il consiste surtout à énoncer si les règles de droit ont été appliquées comme il se doit, à la base des faits qui ont été relevés et étudiés par les tribunaux ou les cours d'appel et, à décider s'il n'est plus possible de débattre devant sa propre instance. Si la Cour de cassation juge que la décision contestée découle d'une bonne application de la loi, elle rejettera le pourvoi. Son rôle n'est ainsi que de scruter les décisions de la cour d'appel selon la loi en vigueur au moment du jugement (Bälz 139).

Comme soutenu par nos analyses, l'affaire Nasr Hâmid Abu Zayd traduit un jeu juridique complexe où s'entrecroisent des référents religieux, mais aussi des lois modernes. La notion de hisba qui reste au centre de la plainte est le résultat de l'entrelacement d'un ancien mode d'arbitrage aboli (tribunaux de statut personnel) et la constitution juridique de l'Égypte moderne.

Sur un plan strictement juridique, le recours auprès de la cour d'appel concernant la requête d'Abu Zayd avait reçu un avis favorable en tant que hisba; et ce, malgré le délaissement des tribunaux de statut personnel par sa révocation suite à la proclamation de la loi 462/1955 et son application (Bälz 143).

Dans un cadre plus général, on remarque que même si la «soumission» aux normes et aux prescriptions est au cœur de la tradition islamique, tous les dispositifs judiciaires dans les sociétés à culture musulmane ont été graduellement modifiés au cours des deux derniers siècles (Dupret 95). On assiste aujourd'hui, dans ces pays, notamment arabomusulmans, à une remodulation des attributions juridictionnelles qui, jusque-là, relevaient de la charia et à une mise en place d'un système de normativité qui gravite autour de la réforme du statut personnel ou de la famille (mariage, divorce, filiation, successions), plus ou moins séculaire et au sein duquel coexistent dans un «équilibre transactionnel» (Berque) institué avec l'islam, droit et religion, tradition et modernité, droits universels et spécificités culturelles et identitaires.

L'adoption et la mise en œuvre de lois codifiées dans ce cadre n'ont cependant pas empêché depuis les années 1970, une tendance à l'« islamisation » d'un droit estimé trop séculier. Loin de concrétiser un retour à la normativité islamique classique, cette tendance s'explique par l'adoption de textes législatifs faisant clairement référence à la loi islamique (charia); celle-ci étant souvent admise en tant que source principale de la législation et particulièrement en Égypte (Dupret 96). La plupart des États contemporains à majorité musulmane ont intégré dans leur constitution, des 
dispositions faisant référence à l'islam ou à la loi islamique. Très souvent, la faisabilité de ces dispositions relève plus de l'ordre du symbolique ou reste cantonnée à certains secteurs, tel le droit de la famille. Ces références religieuses produisent cependant un effet performatif.

On l'aura compris, c'est dans cette tradition juridique renouvelée où s'entremêlent des normes religieuses réinterprétées ou «inventées ${ }^{13}$ que se construit l'affaire Nasr Hâmid Abu Zayd. Paradoxalement, l'intervention du législateur égyptien se retrouve disqualifiée, cette fois non pas par une norme religieuse, mais bien par une interprétation occidentale du droit. "Les entrepreneurs de morales » qui ont déposé la plainte contre Abu Zayd profitent d'un certain flou juridique pour pouvoir mener leurs luttes politiques. Leur stratégie repose sur une notion (hisba) qui est héritée d'un système juridique supposément obsolète, mais qui se retrouve réquisitionnée dans le contexte d'affrontements idéologiques. Les requêtes en hisba sont devenues pour les avocats avoisinant les milieux islamistes, un moyen de poursuivre des luttes intellectuelles. Ces forces politiques mobilisent alors un référent normatif dans un cadre qui lui est extérieur, à savoir celui du droit. Il devient alors outil efficace pour passer à l'acte en réponse à la fiction sociale de la menace terroriste.

La charia a occupé une place privilégiée au sein dans l'histoire égyptienne. Cependant, pour des raisons d'adaptation au monde actuel, sa prise en compte au niveau de la législation égyptienne était demeurée minime, exception faite du droit de la famille, ce qui n'affectait nullement la conformité du droit appliqué à son égard. L'importance accordée à la loi islamique (charia) par le gouvernement égyptien l'était, entre autres, dans le but de contrecarrer la montée de l'islamisme ${ }^{14}$. En effet, c'était le moyen privilégié d'éviter toute influence de la réislamisation telle que portée par un islamisme (Frères musulmans, salafistes, etc.) fermement opposé à tout calquage de normes occidentales, qui constituait, à son sens, une trahison pour l'Islam (Cherkaoui). Le gouvernement égyptien a donc réintroduit certains principes de la charia dans sa législation, faisant ainsi référence à la

\footnotetext{
${ }_{13}$ On peut donc rapprocher ce cas de figure de ce que Hobsbawm appelle la «tradition inventée» : « [Les traditions inventées] semblent appartenir à trois types qui se recoupent: a) celles qui établissent ou symbolisent la cohésion sociale ou l'appartenance à des groupes, des communautés réelles ou artificielles ; b) celles qui établissent ou légitiment des institutions, des statuts ou des relations d'autorité ; c) celles dont le but principal [est] la socialisation, l'inculcation des croyances, des systèmes de valeur et des codes de conduite » (Hobsbawm et Ranger; Hobsbawm). La référence juridique à la charia semble renvoyer à ces trois dimensions à la fois.

14 Sous le président Anouar el-Sadate, en 1980, la Constitution a été amendée de manière à faire de la charia la source principale de la législation égyptienne. Le contexte, en effet, était celui d'une recherche de monopole sur la religion dans le but de l'arracher, en tant qu'outil de légitimation, à l'emprise des Frères musulmans et des salafistes. El-Sadate s'est fait appeler « al raïs al mou'mine » (le président croyant, littéralement) (Haykal 240).
} 
valeur normative de la charia dans sa constitution (Mohamed-Afify). L'islam devient une arme à double tranchant: d'une part, la mobilisation des référents islamiques par les gouvernements en place illustre bien l'instrumentalisation de la religion à des fins politiques; d'autre part, les islamistes ambitionnent d'introduire la référence islamique dans les domaines politiques, sociaux et économiques. Ils utilisent aussi l'islam comme idéologie politique en concurrence avec les autres discours politiques.

Les mouvements radicaux de l'islam politique vont également s'aligner sur l'application de cette référence pour fonder leur contestation du pouvoir et demander que les pratiques gouvernementales soient mises en conformité avec les engagements religieux de l'État.

On se retrouve alors face à un système assez complexe où s'enchevêtre un ordre juridique d'inspiration occidentale et d'ordre normatif, le tout érigé dans le cadre de luttes mobilisant le référent religieux de manière renouvelée. Le politique utilise une forme de langage spécifique - à savoir celle du religieux - dans un contexte qui dépasse l'opposition de valeurs entre deux camps bien définis.

\section{Conclusion : contexte post-colonial et fiction terroriste}

Les rebondissements de l'affaire Nasr Hâmid Abu Zayd culminent avec des menaces terroristes qui obligent le professeur de littérature à s'exiler aux Pays-Bas. À travers l'analyse de la construction judiciaire de cet événement ainsi que du contexte intellectuel l'entourant, il est légitime d'affirmer qu'il complexifie la question de l'exercice de la terreur politique en faisant jouer de multiples enjeux en amont et en aval de l'affaire. L'objet prioritaire de cette recherche était d'identifier quelques déterminants sociaux qui pouvaient expliquer le développement de cette affaire sous ses multiples aspects, par opposition à une mise en récit qui se concentre seulement sur la question du terrorisme et perpétue souvent l'ignorance des dispositifs qui provoquent cette menace ${ }^{15}$.

Deux considérations principales ont été prises en compte pour mieux cerner ce sujet, à savoir les rivalités intellectuelles et l'aspect juridique. Chacun de ces aspects aide à démontrer les différents niveaux d'analyse sociale par lesquels nous pouvons comprendre cet événement. En effet, le point de départ de l'affaire Nasr Hâmid Abu Zayd reste un conflit au sein du monde académique. Les dispositions particulières du champ intellectuel égyptien ont favorisé l'antagonisme qui a opposé Abu Zayd à la commission

\footnotetext{
${ }^{15}$ Cette perception inclut aussi une certaine représentation des sociétés musulmanes comme étant des sociétés intrinsèquement intolérantes. Dans cette vision, l'intolérance se manifeste à travers différents types de menaces (incluant le terrorisme).
} 
chargée d'évaluer sa promotion. Le manque d'autonomie des professions intellectuelles a encouragé la subordination des universitaires à des enjeux éloignés des questions scientifiques. Le contexte autoritaire, participant à idéologiser le discours intellectuel, a rendu tout dialogue difficile. Chaque position par rapport au fait religieux (fondamentaliste, moderniste, réformiste...) est devenue une clôture (Roussillon 47) qui empêche tout rapprochement.

Le procès qu'a subi Abu Zayd est révélateur d'un régime juridique particulier. En effet, la notion de hisba est héritée d'une règle judiciaire de nature religieuse sous régime colonial. La transposition de ce type de norme dans un contexte de post-indépendance provoque une série de contradictions, le tout étant amplifié par le fait que l'État égyptien a tenté de se construire une certaine crédibilité grâce à la symbolique religieuse. Cet état de fait ouvre la porte à une certaine surenchère normative pour justifier des luttes politiques.

Ces deux aspects de l'affaire Nasr Hâmid Abu Zayd permettent d'expliquer l'agencement social particulier qui précède la menace terroriste. En tentant de cartographier les différents enjeux qui motivent cet événement, on constate que ce cas dépasse largement le cadre de la violence politique. Au prisme de cette affaire, l'Égypte semble refléter un État d' «hybridité post-coloniale » (Bhabha) qui nous pousse à reconsidérer les enjeux sociaux qu'elle soulève. La trame explicative religieuse ou bien culturaliste du terrorisme semble alors mal adaptée pour objectiver ces zones intermédiaires. En effet, les États post-coloniaux contemporains sont caractérisés par une certaine confusion face à la loi (religieuse). L'islam prend alors une dimension instrumentale, dont le but premier est de préserver les intérêts de certains groupes. Ceci explique alors pourquoi l'arbitraire qui règne dans plusieurs États majoritairement musulmans est souvent justifié par des référents religieux.

Il semble donc difficile de comprendre le phénomène terroriste comme un élément isolé. Ceci est en opposition avec toute fiction sociale qui ne verrait dans l'affaire Nasr Hâmid Abu Zayd que le résultat d'un archaïsme religieux se manifestant notamment par le recours à la menace terroriste. Face au cadrage médiatique qui simplifie le phénomène terroriste, le chercheur se doit de rétablir la complexité intrinsèque à tout phénomène social. 


\section{Bibliographie}

Abaza, Mona, et Georg Stauth. « Occidental Reason, Orientalism, Islamic Fundamentalism - a Critique ». International Sociology 3.4 (1988), pp. 343-364.

Abdel-Malek, Anouar. L'orientalisme en crise. Paris, France: Revue «Diogène », 1963.

Abderraziq, Ali, et Abdou Filali-Ansary. L'Islam et les fondements du pouvoir. Éditions La Découverte, 1994.

Abu Zayd, Nasr Hamid. Critique du discours religieux. Actes Sud, 1999.

---. Reformation of Islamic Thought: A Critical Historical Analysis. Amsterdam University Press, 2006.

---. Al-Ittijâh Al- Aqlîfi al-Tafsîr: Dirâsa fî Qadiyat al-Majâz fì "l-Qur'ân ind alMù tazila (Le rationalisme dans l'exégèse : une étude du problème de la métaphore dans les écrits des Mutazilites). El markaz el thakafi el arabi, 2003.

---. Falsafat al-Ta'wîl : Dirâsa fi Ta'mî al-Qur'ân ind Mubŷ̀ al-Dîn ibn 'Arabî (La philosophie de l'Herméneutique : Une étude de l'herméneutique du Coran de `Ibn `Arabî). Dar Al Tanwir ,1983.

---. Mafhûm al-Nass: Dirâsa fî̀ "Ulûm al-Qur'ân (Le concept du texte : une étude des sciences coraniques). El markaz el thakafi el arabi, 1998.

---. Ishkâliyât al-Qirầa wa Aliyyât al-Ta'wîl (La problématique de la lecture et de la méthode d'interprétation). El markaz el thakafi el arabi, 1995.

---. Al-Imâm al-Sh fi î wa Ta'sîs al-Aydiyulujizyy al-W asatiyya (La création de l’idéologie modérée dans la pensée islamique par Al-Shafi î). Maktabet Madbouly, 1997.

---. Naqd al-Khitâb al-Dîn̂̀ (Critique du discours islamique). Caire : Sina Nachr, 1994.

---. Al-Mar'a fì Khitbâ al-Ažma (La femme dans le discours de crise). Dar noussous li nachr, 1994.

---. Al-Tafkîr fì Zaman al-Takfîr (Pensée en période d'excommunication). Maktabet Madbouly, 1995.

---. Hakadhâ Takallama Ibn `Arab̂̀ (Ainsi parlait Ibn `Arabi). The Egyptian National Organization for Books, 2002.

Abu Zayd, Nasr Hamid et Zubaida Sami. «The Politics of the Islamic Investment Companies in Egypt». Bulletin (British Society for Middle Eastern Studies) 17. 2 (1990), pp. 152-161.

Abu Zayd, Nasr Hamid et Esther R. Nelson. Voice of an Exile: Reflections on Islam. Greenwood Publishing Group, 2004.

Adams, Charles C. Islam and Modernism in Egypt: A Study of the Modern Reform Movement Inaugurated by Muhammad 'Abduh. Russell \& Russell, 1968.

Arkoun, Mohamed. «Discours islamiques, discours orientalistes et pensée scientifique » in Bernard Lewis), Edmund Leites \& Margaret Case, eds, As Others See Us : Mutual Perception, East and West. Princeton University Press, 1985. 
Ayubi, Nazih N. Political Islam: Religion and Politics in the Arab World. Routledge, 1991.

Bakr, Ayman, Elliott Colla, et Nasr Hamid Abu Zayd. «Silencing is at the Heart of My Case ». Middle East Report 185 (1993), p. 27.

Bälz, Kilian. "Submitting Faith to Judicial Scrutiny through the Family Trial : The "Abu Zayd Case" ». Welt des Islams 37 (1997), pp. 135-155.

Bano, Masooda et Keiko Sakurai, (ed.) Shaping Global Islamic Discourses : The Role of Al-a々har, Al-medina, and Al-mustafa. Edinburgh University Press, 2015.

Benzine, Rachid. Les nouveaux penseurs de l'Islam. Éditions Albin Michel, 2008.

Bernard-Maugiron, Nathalie. "Anatomie sociologique d'une affaire égyptienne. Le procès de "l'Émigré", de Youssef Chahine ", Gilles Boëtsch, Baudouin Dupret \& J.-N. Ferrié, Droits et sociétés dans le monde arabe et musulman: perspectives socioanthropologiques, Aix-en-Provence, PUAM, 1997, pp. 167-192.

Berque, Jacques. Arabies : Entretiens avec Mirese Akar. Stock, 1978.

Bhabha, Homi K. Les lieux de la culture : une théorie postcoloniale. Payot, 2007.

Blair, Sheila S. «A brief biography of Abu Zayd ». Muqarnas, Volume 25. Brill, 2008, pp. 155-176. Web.

Bourdieu, Pierre. Le Sens Pratique. Les éditions de minuit, 1980.

Cahen, Claude, et Talbi Mohamed. "Hisba». Encyclopedie de l'islam 2. Leiden : E. J.Brill, 111 (1989), pp. 503-505.

Campanini, Massimo. "Qur'an and Science : A Hermeneutical Approach/القر آنو العلم: منهجتفسيري. Journal of Qur'anic Studies (2005), pp. 48-63.

Cherkaoui, Mohamed. Le Paradoxe des conséquences. Librairie Droz, 2015, pp. 165-185.

Demers, François. «Journalistes et communicateurs au temps de

l'information existentielle ». Communication et organisation 8 (1995):

Communicationorganisation.revues.org. Web.

De Mijolla-Mellor, Sophie, et Adam Kiss. «Avant-propos ». Topique 83 (2003), p. 5.

Dupret, Baudouin, éd. La charia aujourd'bui: usages de la référence au droit islamique. Éditions La Découverte, 2012.

Fayek, Maha. « 5 - Egypte : une presse qui tue ». Cahiers libres (2016), pp. 98112.

Ferrié, Jean-Noël, et Baudouin Dupret. « Participer au pouvoir, c'est édicter la norme : sur l'affaire Abu Zayd (Égypte, 1992-1996)». Revue française de science politique 47.6 (1997), pp. 762-775.

Filali-Ansary, Abdou. «Ali Abderraziq et le projet de remise en ordre de la conscience islamique ». Égypte/Monde arabe 20 (1994), pp. 185-201.

Flahault, François, et Nathalie Heinich. "La fiction, dehors, dedans ». L'Homme. Revue française d'anthropologie 175-176 (2005), pp. 7-18. 
Gamson, William A., et Andre Modigliani. « Media Discourse and Public Opinion on Nuclear Power: A Constructionist Approach ». American Journal of Sociology 95.1 (1989), pp. 1-37.

Goffman, Erving. Frame Analysis : An Essay on the Organization of Experience. Harvard University Press, 1974.

Haykal, Muhammad Hasanayn. Autumn of Fury : The Assassination of Sadat. Random House, 1983.

Heinich, Nathalie, et Jean-Marie Schaeffer. Art, création, fiction : entre philosophie et sociologie. Éditions Jacqueline Chambon, 2004.

Hobsbawm, Eric. «Inventer des traditions ». Trad. par André Mary, Karim Fghoul, et Jean Boutier. Enquête. Archives de la revue Enquête 2 (1995), pp. 171-189. Web.

Hobsbawm, Eric, et Terence O. Ranger. The Invention of Tradition. Cambridge University Press, 1992.

Hume, David, et Michel Malherbe. Enquête sur l'entendement humain. J. Vrin, 2008.

Johansen, Baber. "Apostasy as objective and depersonalized fact : two recent Egyptian court judgments ». Social Research (2003), pp. 687-710.

Kahneman, Daniel, et Amos Tversky. «Prospect Theory : An Analysis of Decision under Risk ». Econometrica 47.2 (1979), pp. 263-291. Web.

Kant, Emmanuel. Critique de la faculté de juger. Flammarion, 2015.

Kepel, Gilles. Le Prophète et Pharaon: Aux sources des mouvements islamistes. Nouvelle. Seuil, 1993.

Lejeune, Philippe. Signes de vie. Le pacte autobiographique 2 : Le Seuil, 2015.

Lits, Marc. Du 11 septembre à la riposte : les débuts d'une nouvelle guerre médiatique. Bruxelles : De Boeck, 2004.

Mohamed-Afify, Ayman. « La Constitution égyptienne de 2014: entre traditions et tendances révolutionnaires ». Revue française de droit constitutionnel 101 (2015), pp. 121-144.

Najjar, Fauzi M. «Islamic Fundamentalism and the Intellectuals : The Case of Nasr Hamid Abu Zayd ». British Journal of Middle Eastern Studies 27.2 (2000), pp. 177-200.

Noam, Eli, et Eric Brousseau. «La déréglementation de la télévision va-telle renforcer la domination d'Hollywood? Une critique économique d'un raisonnement traditionnel ». Réseaux 9.50 (1991), pp. 159-172. Web.

Passeron, Jean-Claude, et Jacques Revel. Penser par cas. Éditions de l'École des Hautes Études en Sciences Sociales, 2005.

Roussillon, Alain. La pensée islamique contemporaine : Acteurs et enjeux. Téraèdre, 2005.

Said, Edward W. L'orientalisme: l'Orient créé par l'Occident. Seuil, 2005.

Scheufele, Dietram A. «Framing as a theory of media effects ». Journal of communication 49.1 (1999), pp. 103-122. 
Sfeir, George N. «Basic freedoms in a fractured legal culture : Egypt and the case of Nasr Hamid Abu Zayd ». The Middle East Journal (1998), pp. 402-414.

Taji-Farouki, Suha. Modern Muslim Intellectuals and the Qur'an. OUP, 2006.

Temlali, Yassine. " Entretien avec Nasr Hamed Abou Zeïd ». www.babelmed.net. N.p., s. d. Web.

Temlali, Yassine. «La censure religieuse en Egypte: Al-Azhar et le droit de « Hisba » ». www.babelmed.net. N.p., s. d. Web.

Tétu, Jean-François. "Pour une Critique des Médias ». Questions de communication 10 (2006), pp. 247-262. Web.

Thielmann, Jörn. «La jurisprudence égyptienne sur la requête en Hisba ». Égypte/Monde arabe 34 (1998), pp. 81-98.

Zubaida, Sami. «The Politics of the Islamic Investment Companies in Egypt ». Bulletin (British Society for Middle Eastern Studies) 17. 2 (1990), pp. 152161. 